\title{
Winter Rye Cover Cropping Changes Squash (Cucurbita pepo) Phyllosphere Microbiota and Reduces Pseudomonas syringae Symptoms
}

\author{
Rémi Maglione, ${ }^{1,2}$ Marie Ciotola, ${ }^{1}$ Mélanie Cadieux, ${ }^{1}$ Vicky Toussaint, ${ }^{1}$ Martin Laforest, ${ }^{1, \dagger}$ and Steven W. Kembel ${ }^{2, \dagger}$ \\ ${ }^{1}$ Research and Development Centre, Agriculture and Agri-Food Canada, Saint-Jean-sur-Richelieu, QC, Canada \\ ${ }^{2}$ Département des sciences biologiques, Université du Québec à Montréal, QC, Canada
}

Accepted for publication 17 September 2021.

\section{ABSTRACT}

Cover cropping is a soil conservation practice that may reduce the impacts of the economically important pathogen Pseudomonas syringae on crops, including squash (Cucurbita pepo). To date, no studies have directly quantified the effect of rye cover crops on $P$. syringae populations or on the bacterial community of squash leaves. In this work, we tested the hypothesis that the protective effects of cover cropping on squash may be mediated by cover cropping effects on the plant's microbiota that, in turn, protect against $P$. syringae. Using combined $16 \mathrm{~S}$ sequencing and culture-based approaches, we showed that rye cover cropping protects squash against $P$. syringae, by decreasing pathogen population size on squash leaves and increasing fruit health and marketability at harvest. We also found evidence of a strong effect of rye cover crops on bacterial communities of the squash phyllosphere. Those findings were more striking early in the growing season. Finally, we identified numerous phyllosphere bacteria belonging to the genera Sphingomonas, Methylobacterium, and Pseudomonas that were promoted by rye cover crops. Overall, our findings suggest that cover cropping is effective for the sustainable management of $P$. syringae on squash and may provide a reservoir of potential microbial biocontrol agents colonizing the phyllosphere.

Keywords: agriculture, metagenomics, microbiome, plant pathology, rhizosphere and phyllosphere
Cover cropping, or the growth of a plant to cover the soil for environmental benefits rather than for its harvest, is an increasingly popular option available to farmers to address the environmental and human health challenges associated with agricultural intensification (Fawell and Nieuwenhuijsen 2003). Cover cropping allows equivalent yield ( $\mathrm{Lu}$ et al. 2000) or an increase in yield (Fawcett et al. 2015, 2017; Stirzaker and White 1995), weed control (Teasdale 1996), and nematode control (Hooks et al. 2010), and

†Corresponding authors: S. W. Kembel; kembel.steven_w@uqam.ca, and M. Laforest; martin.laforest@ canada.ca

Funding: Support for this project was provided by Agriculture and Agri-Food Canada R\&D Grant (projects J-001235 and J-001785) (to V. Toussaint, M. Laforest, M. Ciotola, and S. W. Kembel), and by a Natural Sciences and Engineering Research Council of Canada Discovery Grant (to S. W. Kembel) and Canada Research Chair (S. W. Kembel).

*The $\boldsymbol{e}$-Xtra logo stands for "electronic extra" and indicates that supplementary materials are published online.

The author(s) declare no conflict of interest. reduces soil erosion (Dabney et al. 2001; Stirzaker and White 1995). Winter cover crops used in northern countries are a promising avenue to reduce soil erosion and depletion by covering the soil during the winter (Dabney et al. 2001). Cover crops can also improve water quality by reducing herbicide runoffs (Hall et al. 1984), and improve soil condition by reducing temperature variations and water loss (Teasdale and Mohler 1993). Many studies have demonstrated the benefits of cover cropping, although there is controversy about potential negative effects of cover cropping such as the potential transfer of pathogens from cover crop to crop (Bakker et al. 2016). Cover cropping is known to shape the soil microbiome (Hartman et al. 2018) but, to date, no study has quantified cover crop effects on aboveground microbial communities.

The phyllosphere microbiome (the microbial communities of aboveground plant parts, particularly leaves) is composed of a broad range of microorganisms such as bacteria, viruses, fungi, and archaea (Lindow and Brandl 2003). The microbiota on the aboveground parts of plants can improve plant fitness and biomass, primarily by reducing pathogen symptoms thanks to direct competition or associated with plant volatile compound (Abanda-Nkpwatt et al. 2006; Ritpitakphong et al. 2016). Microorganisms are also important pathogens of the phyllosphere: 20 to $30 \%$ 
of crop production losses worldwide are due to various pests and pathogens (Savary et al. 2019), and microbial pathogens are estimated to account for $16 \%$ of potential losses (Oerke 2006). Pseudomonas syringae, one of the most widely studied bacterial plant pathogens, can infect a wide range of host plants, including many economically important crops; it begins life as a leaf epiphyte, colonizes the host apoplast through wounds and stomates, and then damage fruit (Hirano and Upper 2000; Xin et al. 2018). Long-term intensive and frequent monocropping favors emergence of local pathogenic P. syringae reservoirs (Lindemann et al. 1984). Efforts have been made to biologically control this pathogen on leaves using microbial competition; Lindemann and Suslow (1987) used a competition population of $P$. syringae disarmed with an ice nucleation mutation to prevent pathogen-related frost damage on strawberry plants and Innerebner et al. (2011) used several Sphingomonas spp. on leaf surfaces to protect Arabidopsis thaliana plants from $P$. syringae. Moreover, many studies of plant induced systemic resistance have noted potential leaf control of $P$. syringae based on the microbe-associated molecular patterns mechanism and, interestingly, via microbial competitors located in the root-associated microbiome (Pieterse et al. 2014; Van Wees et al. 2008). Finally, prior colonization of the plant phyllosphere by the beneficial bacterium is a well-known strategy to preemptively prevent the growth of the pathogen (Andrews 1992).

Despite the potential for microbiota-based biological control of $P$. syringae, management of this pathogen is primarily done through copper application, which has led to the development of resistance (Bender and Cooksey 1986). Past studies have suggested that cover crops may provide soilborne biological control of pathogens (Abawi and Widmer 2000; Collins et al. 2006), and we have found that rye (Secale cereale) cover crops (RCC) helped to reduce $P$. syringae symptoms incidence on squash leaves (V. Toussaint, personal communication). The mechanism of this protective effect of RCC against squash bacterial leaf spot is not known; however, we hypothesize that it may be mediated by cover cropping effects on the plant's microbiota that, in turn, protect against $P$. syringae.

In this study, we used sequence- and culture-based approaches to quantify the effects of different cover cropping approaches on bacterial communities on squash leaves infected by $P$. syringae. We first evaluated whether cover crops could help to reduce $P$. syringae populations on squash leaf surfaces by direct measurement of pathogen abundance on leaves. We also considered the fruit's health and marketability at harvest in such cropping practices. We then estimated the effects of different cover cropping methods on phyllosphere bacterial communities by quantifying leaf microbiome diversity and composition using a bacterial metabarcoding approach. Finally, we identified the bacterial taxa that were most strongly influenced by cover cropping practices.

\section{MATERIALS AND METHODS}

Experimental design and field treatment. All samples in this study were collected from the Agriculture and Agri-Food Canada L'Acadie Experimental Farm at Saint-Jean-sur-Richelieu, Quebec, Canada $\left(45^{\circ} 17^{\prime} 48.7^{\prime \prime} \mathrm{N}, 7^{\circ} 20^{\prime} 14.8^{\prime \prime} \mathrm{W}\right)$. The experiment comprised six replicates of four cover cropping treatments in a fully randomized block design, with a total of 24 plots containing three raised beds with a single line of squash each (Supplementary Fig. S1). The cover cropping treatments were rye cover crop (RCC), chemically terminated rye cover crop (CT-RCC), plastic cover (PC), and bare soil (BS). Specifically, for the 2016 and 2017 growing seasons, the RCC treatment consisted of fall rye ('Gauthier' cultivar) seeded at a rate of $250 \mathrm{~kg} / \mathrm{ha}$ on 14 September 2015 and 16 September 2016 and rolled to the ground the following spring by crimping rye with a three-sectional roller crimper (I\&J Mfg., PA, U.S.A.) on 13 June 2016 and 2017 (Supplementary Fig. S2 for crimping process); the CT-RCC treatment was the same as RCC except rye was killed with a herbicide (glyphosate, Roundup, WeatherMax; Bayer) at a rate of $2.16 \mathrm{~kg}$ acid equivalent $\mathrm{ha}^{-1}$ ) before the rye crimping on 10 June 2016 and 8 June 2017; the PC treatment consisted of the application of an agricultural plastic mulch over each raised bed within plots; and the BS treatment was a bare soil mound lane that did not receive any cover cropping treatment. All of the soil was covered by rye and most of the cover crop material remained on the soil during the growing season and, except for a change in color, no significant decay was observed (Supplementary Fig. S2). Squash seeding was performed on 15 June 2016 and 2017; mechanically with a seeder for the BS, RCC, and CT-RCC and manually for the PC (Supplementary Fig. S2). To monitor treatment effects on pathogen populations, we inoculated squash seeds with a rifampicin-resistant $P$. syringae strain prior to direct seeding into raised beds. Although plants are frequently naturally inoculated by seedborne or soil pathogen reservoirs, we needed to ensure potential infection to be able to quantify the effects of the cover cropping treatments. Strain pathogenicity was confirmed by hypersensitive reaction testing in tobacco leaves and seed inoculation was validated by growing on-field negative control plants at the border of each treatment plot. Control plants were asymptomatic during the entire growing season.

Microbial collection, DNA extraction, and sequencing. Microbial communities of the phyllosphere were collected from squash at three different times during each growing season in 2016 (12 July, 1 August, and 1 September 1) and 2017 (12 June, 31 July, and 5 September), defined as Early, Mid, and Late season, respectively. Each sample consisted of a mix of young and old leaves harvested from the squash canopy by clipping an average of $16.8 \pm 8.3$ and $20.7 \pm 4.8 \mathrm{~g}$ of leaves for years 2016 and 2017, respectively (Supplementary Table S1) from an individual plant into sterile sample bags (SCR-7012-ID; Innovation Diagnostics Inc., Blainville, QC, Canada) with surface-sterilized shears. Replicate samples (three per plot) were collected for a total of 72 samples per sampling date (three samples by four treatments by six replicates). Microbial cells were then gathered by washing each leaf sample using with $110 \mathrm{ml}$ of saline buffer $(0.85 \% \mathrm{NaCl})$ and using a homogenizer blender (Stomacher 400; Seward, U.K.) for $30 \mathrm{~s}$ at $250 \mathrm{rpm}$.

A volume of $1 \mathrm{ml}$ of wash solution from each sample was placed on King's B medium with cycloheximide (50 mg/liter) (C7698; Sigma-Aldrich, Oakville, ON, Canada) and rifampicin (50 mg/liter) (R3501; Sigma-Aldrich), allowing us to estimate $P$. syringae population size by counting CFU after 4 days of growth at $28^{\circ} \mathrm{C}$.

The remaining $100 \mathrm{ml}$ of wash solution was divided into two 50-ml Falcon tubes; one was centrifuged at 11,500 $\times g$ for $20 \mathrm{~min}$ and the other at $4,500 \times g$ for $20 \mathrm{~min}$. The aqueous phase was removed from both tubes and the pellet in the Falcon tube centrifuged at $4,500 \times g$ was frozen at $-80^{\circ} \mathrm{C}$. The DNA of the remaining pellet was extracted using MoBio PowerSoil DNA extraction kits (CA-11011-418; VWR, Mont-Royal, QC, Canada) and stored at $-20^{\circ} \mathrm{C}$ for future processing. Amplicon libraries were prepared for Illumina sequencing using PCR targeting the V5-V6 region of the bacterial $16 \mathrm{~S}$ ribosomal RNA gene using cyanobacteriaexcluding primers (16S primers 799F-1115R) (Chelius and Triplett 2001; Redford et al. 2010) to exclude chloroplast DNA (for a $16 \mathrm{~S}$ amplicon structure overview, see Supplementary Fig. S3). The $25-\mu \mathrm{l} \mathrm{PCR}$ assays consisted of $5 \mu \mathrm{l}$ of $5 \times$ HF buffer (Thermo Scientific, Waltham, MA, U.S.A.), $0.75 \mu$ of dimethyl sulfoxide, $0.5 \mu \mathrm{l}$ of dNTPs (10 mM each), $0.25 \mu \mathrm{l}$ of Phusion Hot Start II polymerase (Thermo Scientific), $1 \mu \mathrm{l}$ of each primer $(5 \mu \mathrm{M}), 1 \mu \mathrm{l}$ of genomic DNA, and $15.5 \mu \mathrm{l}$ of molecular-grade water (IDT, Coralville, IA, U.S.A.). We included a negative control ( $1 \mu$ l of sterile water; 
IDT) as well as a positive control ( $1 \mu \mathrm{l}$ of $P$. syringae DNA) in each 96-well library plate. Libraries were checked on agarose gels $(2 \%)$, normalized with SequalPrep kit (A1051001; Life Technologies, Burlington, ON, Canada) on a Gilson robot (Middleton WI, U.S.A.) and sequenced on MiSeq (Illumina, San Diego, CA, U.S.A.). For each year, samples were randomly assigned between two sequencing runs, representing a total of four runs.

Sequence analysis. Sequencing adaptors were removed with the bbduk tool from bbmap (38.86, https://sourceforge.net/projects/ bbmap/), with the following parameters: $\mathrm{ktrim}=\mathrm{r}, \mathrm{k}=23$, mink $=$ 11 , hdist $=1$ tpe tbo (Bushnell 2014). Sequences were thereafter demultiplexed, allowing one mismatch on the barcode sequence with deMulMe (https://github.com/RemiMaglione/genomicScript/ tree/master/deMulMe). Sequence barcodes were removed with cutadapt 2.10 in paired-end mode (Martin 2011). In total, we obtained $11,929,677$ and 11,816,138 demultiplexed paired-end sequences for 2016 and 2017, respectively. All subsequent data processing and computations were done with DADA2 1.12.1 (Callahan et al. 2016) in $\mathrm{R}$ 3.6.0 ( $\mathrm{R}$ Core Team 2013) and graphs were produced using the ggplot2 3.2.1 package (Wickham 2016). Sequences were trimmed and quality filtered with filterAndTrim with default parameters, except trimLeft $=c(19,26)$, truncLen $=c(230,210)$, and maxEE $=\mathrm{c}(2,3)$; trimLeft was set to remove PCR primers used for library preparation, and truncLen and maxEE were set to yield filtered sequences with a quality around a phred score of 30. Amplicon sequence variants (ASVs) were constructed from filtered sequences with the following set of built-in DADA2 functions and their default parameters, except as mentioned: dada in pseudopooling mode, mergePairs with minOverlap $=30$, collapseNoMismatch with minOverlap $=240$, and removeBimeraDenovo with method = "pooled". ASVs were then taxonomically annotated by assignTaxonomy with the SILVA version 128 database (Quast et al. 2013; Yilmaz et al. 2014). For 2016 and 2017 data, the DADA2 pipeline yielded a mean of 10,484,056 filtered paired-end sequences used to identify 7,604 ASVs, which represent an average of 29,652 sequences and $165 \mathrm{ASVs}$ per sample.

A preliminary evaluation of control samples was performed by comparing the composition of control and phyllosphere samples using a principal component analysis ordination of a distance matrix obtained with centered log ratio (clr) transformation of the original community matrix (Gloor 2016). Because negative control samples would be lost by excluding samples with very few sequences, the clr transformation allowed us to keep all of the samples while identifying outlier samples. Because the positive and negative control samples were distinct compositionally from the phyllosphere samples (Supplementary Fig. S4), they were removed from all further analyses. Positive control samples were examined and determined to be dominated by ASVs corresponding to the expected mock community species composition.

Data analysis. P. syringae abundance analysis on squash leaves. $P$. syringae count differences between treatments were evaluated with a linear mixed model of treatment effects on $P$. syringae abundance for 2016 and 2017 data. Effects of experimental blocks were integrated as a random effect. Effect of treatment was estimated with a Tukey's honestly significant difference (HSD) posthoc test performed on the abovementioned model.

Squash fruit health and marketability at harvest. We quantified squash fruit health and marketability by harvesting fruit within a 10by-10-m area within each plot. Fruit health and marketability was determined with four categories of $P$. syringae symptoms based on the visually estimated proportion of fruit affected by the considered symptoms: $P$. syringae symptoms outside of the fruit, $P$. syringae symptoms that penetrated the fruit, $P$. syringae symptoms that left a scar at the surface of the fruit, and $P$. syringae symptoms that generated squash rot. Marketability was assessed based on these categories of $P$. syringae symptoms, where $>1 \%$ of fruit affected in at least one category prevents marketability. This cut-off was chosen to address the actual market plasticity, where squash fruit with low symptoms can still be sold. Healthy fruit was defined as a squash fruit with no $P$. syringae symptoms. Thus, marketability and fruit health were binomially distributed and their differences among treatments were evaluated with a generalized mixed linear model, where blocking effect was integrated as random variable, for both years. Effects of treatment on fruit health and marketability were estimated with a Tukey's HSD posthoc test (using the glht function of multcomp $\mathrm{R}$ package) performed on the abovementioned model.

Effect of cover cropping treatments on bacterial community diversity. Diversity analyses were performed using the $\mathrm{R}$ package phyloseq 1.30.0 (McMurdie and Holmes 2013), picante 1.8.1 (Kembel et al. 2010), and vegan 2.5-6 (Oksanen et al. 2007). To evaluate the effect of treatments on community diversity on squash leaves, samples were randomly rarefied to 5,000 sequences per sample; this threshold was chosen to preserve the maximum number of samples with a sufficient quantity of ASVs to capture the majority of the diversity in each sample (Supplementary Figs. S5 and S6). For all diversity analyses, rarefactions and their subsequent analyses were repeated 1,000 times but no qualitative differences were observed between iterations; therefore, we report here the results of a single random rarefaction of the data. The uniformity of relative abundance distributions of ASVs ( $\alpha$ diversity) was assessed with the Shannon index (Haegeman et al. 2013). The effect of treatment on $\alpha$ diversity was evaluated with a posthoc test (Tukey's HSD) of a linear model ( $\alpha$ diversity as a function of treatment). Variation in bacterial community structure among samples was quantified with the Bray-Curtis index (Bray and Curtis 1957). Major gradients in community composition were evaluated with nonmetric multidimensional scaling (NMDS) ordination of weighted BrayCurtis distances among samples. We partitioned the variance in phyllosphere bacterial community structure explained by sampling date and treatment using permutational multivariate analysis of variance (PERMANOVA) of the variance in Bray-Curtis dissimilarities, and linear mixed models of sample scores on the NMDS ordination axes. We evaluated whether treatments were associated with compositionally distinct groups of samples with a least squares comparison (emmeans v1.4.8 R package) (Lenth et al. 2018) of a linear mixed model with NMDS axis scores as a function of treatment (fixed effect) and with experimental block as random effect.

Differential abundance analysis of ASVs. Differential abundance analysis of ASVs among treatments were performed with DeSeq2 3.11 (Love et al. 2014). The ASV matrix was filtered using the CoDaSeq R package 0.99.4 (Gloor 2016), with the codaSeq.filter function with the following parameters: min.reads $=1,000$ (minimum reads per sample), min.prop $=0.00001$ (minimum proportional abundance of a read in any sample), and min.occurrence $=$ 0.005 (minimum fraction of nonzero reads for each variable in all samples). Because DeSeq2 takes nonzero positive integers as input, we transformed the abundance matrix to pseudocounts by adding 1 to each cell in the matrix prior to analysis (Nearing et al. 2021). DeSeq2 analysis was executed with parameters recommended for single-cell analysis that better fit data with a zero-inflated negative binomial distribution such as our community matrix. We tested for differential abundance by contrasting ASV abundances across all six possible treatment comparisons: RCC versus CT-RCC, RCC versus PC, RCC versus BS, CT-RCC versus PC, CT-RCC versus BS, and $\mathrm{PC}$ versus BS. We used the following model: design $=\sim$ block + treatment and the blocking random variable was controlled through the reduced parameter. Only contrasts with adjusted $P$ value $\leq 0.01$ and $\log _{2}$-fold-change $\geq 1$ were considered to be significantly 
differentially abundant. Taxonomic annotations were set at the genus level for the differential abundance analysis of ASVs. Phylogenetic trees have been built with Fastree2 2.1.3 (Price et al. 2010) on ASV sequence alignments with QiiME1 (PyNAST as default method) (Caporaso et al. 2010a, b).

\section{RESULTS}

Cover cropping reduced $\boldsymbol{P}$. syringae abundance on squash leaves and improved fruit health and marketability. We found that $P$. syringae was less abundant on the leaves of squash grown with RCC (Fig. 1) and harvested squash fruit were more marketable and healthier with RCC treatments (Table 1). In 2016, P. syringae population size was significantly lower for the RCC treatment compared with PC and BS treatments during the Early season (Tukey's HSD posthoc on linear model) (Fig. 1). There were no significant differences among treatments during Mid and Late season sampling in 2016. On the other hand, in 2017, P. syringae population size was significantly lower during the Early season for both RCC treatments (RCC and CT-RCC) compared with PC and BS treatments (Tukey's HSD posthoc test on linear mixed model) (Fig. 1). No
$P$. syringae colonies were retrieved at Mid season on the squash leaves grown with RCC, and pathogen populations were lower for CT-RCC as compared with PC and BS treatments. Finally, pathogen viable cells counts were significantly lower in RCC as compared with BS at Late season sampling. Taken together, $P$. syringae populations showed the greatest reduction in the RCC treatments early in the growing season. This result was consistent during both years (Supplementary Tables S2 and S3). Moreover, fruit health and marketability were significantly different across all years of harvest $(P<0.05$, Tukey's HSD). Indeed, marketability with RCC was significantly different from BS and PC. Average marketability with RCC was $13 \%$ higher in 2016 and $6.2 \%$ higher in 2017 as compared with BS, and 8\% higher in 2016 and $4.3 \%$ higher in 2017 as compared with PC (Table 1). Fruit health increased 14\% in 2016 with RCC treatment as compared with BS and 13\% in 2017 with CT-RCC treatment as compared with BS (Table 1). No further significant differences among treatments were observed for fruit health and marketability in both years of harvest.

Phyllosphere microbial communities differed between sampling dates and treatments. Cover cropping treatments influenced bacterial community composition on squash leaves. Treatments also

\section{6}
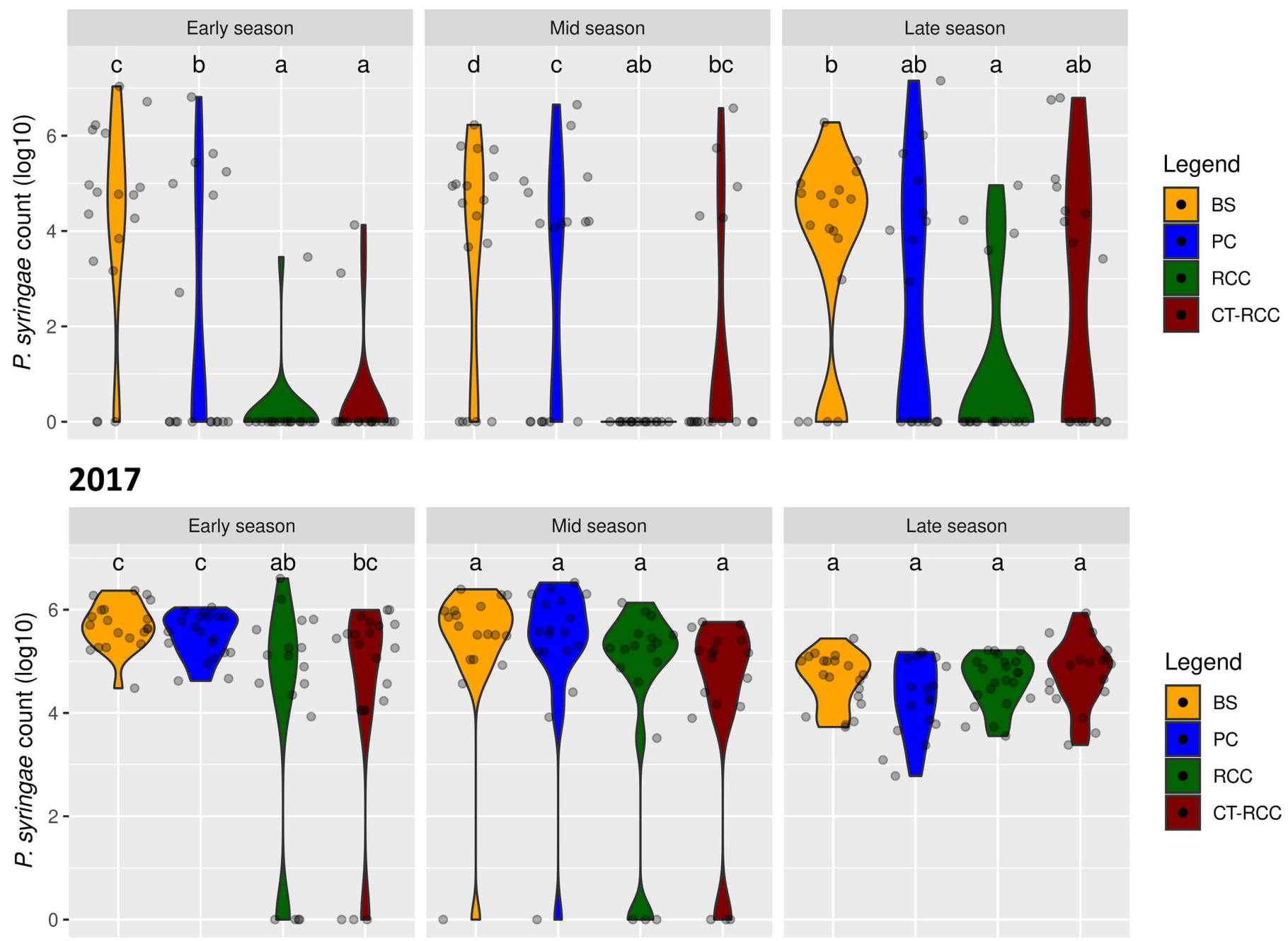

Fig. 1. Pseudomonas syringae populations on squash leaves for different cover cropping practices during 2016 and 2017. Squash pathogen population sizes were estimated based on CFU count from bacterial culture of each leaves sample retrieved from four cropping treatments: bare soil (BS), plastic cover (PC), rye cover crop (RCC), and chemically terminated rye cover crop (CT-RCC). Different letters represent significantly different treatments $(P<0.05)$ from a posthoc test (Tukey's honestly significant difference) of a linear model ( $P$. syringae as a function of treatment) in 2016 or a linear mixed model ( $P$. syringae as a function of treatment [fixed effect] and block [random effect]) in 2017. 
affected bacterial diversity and richness (Supplemental Analysis 1 with Supplementary Figs. S7 and S8). NMDS ordination of the overall community distance matrix suggests that squash phyllosphere samples clustered by sampling dates in both years (Supplementary Fig. S9); sampling date accounted for $28 \%\left(R^{2}=0.28, P<\right.$ $0.001)$ and $11 \%\left(R^{2}=0.11, P<0.001\right)$ of community compositional variation between samples for 2016 and 2017, respectively (PERMANOVA on Bray-Curtis distances among samples). Because there was an interaction between sampling date and treatments (PERMANOVA on Bray-Curtis distances among samples; sampling date-cover crop treatment interaction $P<0.001$ both years), and sampling date accounted for the majority of the effect, we analyzed the effect of treatments on communities separately for each date in order to summarize these complex effects.

Cover cropping treatments influence squash phyllosphere community diversity and composition. Bacterial community $\alpha$ diversity was significantly different among treatments for several sampling dates (linear model; Shannon index versus cover cropping treatment, $P<0.05$ ) (Fig. 2). Bacterial community $\alpha$ diversity was higher for both rye treatments as compared with BS and PC treatments in 2016 in the Early season sampling (Tukey's HSD on linear model, $P<0.05$ ) (Fig. 2). No further differences were observed between treatments at the other sampling dates in 2016. In 2017, although both RCC practices (RCC and CT-RCC) resulted in significantly lower $\alpha$ diversity in Mid season sampling as compared with $\mathrm{BS}$, their $\alpha$ diversity was higher as compared with PC treatments in Early season sampling. No further differences were observed between treatments in Late season sampling of 2017. Taken together, $\alpha$ diversity increased early in the growing season for both RCC treatments (RCC and CT-RCC) as compared with BS and PC treatments of 2016 or to the PC treatment of 2017.

Community composition varied among cover cropping treatments for each sampling date of 2016 and 2017 (PERMANOVA on Bray-Curtis distances for each sampling date; cover cropping effect $P<0.001$ ). Moreover, distances between treatment clusters visible in the ordination (Fig. 3) suggests that treatment effects were more important for Early season sampling as compared with the other sampling dates. Differences in community composition among treatments were more pronounced in Early (PERMANOVA on Bray-Curtis distances; effect of cover cropping treatment $\mathrm{P}_{2016}$ and $\left.2017<0.001 ; R_{2016}^{2}=0.24, R_{2017}^{2}=0.31\right)$ rather than Mid $\left(\mathrm{P}_{2016}\right.$ and $\left.2017<0.001 ; R^{2}{ }_{2016}=0.14, R^{2}{ }_{2017}=0.30\right)$ and
Late $\left(\mathrm{P}_{2016}\right.$ and $\left.2017<0.001 ; R^{2}{ }_{2016}=0.07, R^{2}{ }_{2017}=0.16\right)$ season sampling (Fig. 3).

Ordination of samples based on community composition also indicated that samples clustered into two compositionally distinct groups: RCC and CT-RCC versus PC and BS (Fig. 3). At each sampling date, sample scores on the first axis of the ordination differed significantly among treatments (linear mixed model with NMDS axis scores as a function of treatment [fixed effect] with experimental block as random effect). RCC and CT-RCC were different from the PC and BS but not different from each other (least squares comparisons on the above linear mixed model; Supplementary Fig. S10). Moreover, these two compositionally distinct groups (RCC and CT-RCC versus PC and BS) are more different during the Early season as compared with every other sampling date: these community compositional differences remained throughout the growing season, although they progressively decreased in magnitude (least squares comparisons intervals between these two groups are closer throughout the growing season on the first axis; Supplementary Fig. S10). However, PC and BS remained different in Mid season in 2017 and were often separated along the second axis of the NMDS ordination (Fig. 3) (estimated marginal means test of ordination axis scores; Supplementary Fig. S10).

Sphingomonas and Methylobacterium were more abundant with cover crop treatments. An analysis of differential abundance of ASVs among treatments and sampling dates identified several ASVs that were more abundant in certain treatments and at certain times. As mentioned previously, the abundance of $P$. syringae and the squash phyllosphere communities were influenced by the sampling date; thus, the cover crop effect on taxa abundance was analyzed separately for each sampling date. To identify ASVs that were strongly associated with different cover cropping systems, we took the top differentially abundant ASVs with the highest $\log _{2^{-}}$ fold change in abundance for each treatment comparison (Fig. 4, 2017; Supplementary Fig. S11, 2016). Different cover cropping treatments had several differentially abundant ASVs with $\log _{2}$-fold changes in abundance between treatments ranging from -10.2 to 9.7. Overall, the contrasts of RCC versus CT-RCC and PC versus BS consistently had few and weakly differentially abundant ASVs. Conversely, contrasts of both RCC and CT-RCC treatments versus $\mathrm{PC}$ and BS exhibited more and strongly differentially abundant ASVs. In 2016, ASVs that were significantly more abundant for RCC and CT-RCC treatments included those annotated at the genus

TABLE 1

Proportion of squash fruit (mean \pm standard deviation) with no Pseudomonas syringae symptoms and marketable fruit with no damage for the two growing seasons (2016 and 2017)

\begin{tabular}{|c|c|c|c|c|}
\hline Year & \multicolumn{4}{|c|}{ Proportion of squash fruit (\%) } \\
\hline \multicolumn{5}{|c|}{ No symptoms ${ }^{w}$} \\
\hline 2017 & $74.0 \pm 13.0 \mathrm{ab}$ & $73.2 \pm 7.8 \mathrm{ab}$ & $78.7 \pm 12.1 \mathrm{a}$ & $65.7 \pm 7.7 b$ \\
\hline \multicolumn{5}{|c|}{ No damage ${ }^{y}$} \\
\hline 2017 & $91.2 \pm 7.2 b$ & $95.5 \pm 3.4 \mathrm{a}$ & $94.2 \pm 3.0 a^{2}$ & $89.3 \pm 3.0 \mathrm{~b}$ \\
\hline \multicolumn{5}{|c|}{$\begin{array}{l}\text { " Differences among treatments were tested using Tukey's honestly significant difference (HSD) test, based on a generaliz } \\
\text { general linear hypotheses provided by glht function of multcomp package. Within each year, treatments that do not } \\
\text { significantly different according to the Tukey's HSD test }(P<0.05) \text {. } \\
\text { w Proportion of squash fruit without } P \text {. syringae symptoms. } \\
\text { x Difference between chemically terminated rye cover crop (CT-RCC) and bare soil was marginally significant }(P=0.057) \text {. } \\
\text { y Proportion of marketable squash fruit with no damage. } \\
\text { z Difference between CT-RCC and bare soil was marginally significant }(P=0.064) \text {. }\end{array}$} \\
\hline
\end{tabular}


level as Rhizobium, Pseudomonas, and Saccharibacillus during the Early season, and Chryseobacterium and Sphingomonas during the Mid season. Conversely, ASVs that were significantly more abundant in BS and PC treatments included those annotated as Pseudarthrobacter during the Early season, Exiguobacterium and Pseudathrobacter during the Mid season, and Deinococcus during the Late season. In 2017, ASVs that were significantly more abundant for RCC and CT-RCC treatments included those annotated as the genera Sphingomonas, Methylobacterium, or Hymenobactrium during the Early season; Sphingomonas, Methylobacterium, Aureimonas, and Microbacterium during the Mid season; and Chryseobacterium and Rhizobacterium during the Late season. On the other hand, ASVs that were significantly more abundant in BS and PC treatments included Massila and Exiguobacterium at Early season; Massila, Exiguobacterium, Hymenobacter, Deinococcus, and Pseudarthrobacter at Mid season; and Deinococcus and Microbacterium at Late season.

\section{DISCUSSION}

RCC reduced the abundance of $P$. syringae on squash leaves, improved the health and marketability of fruit, and shaped phyllosphere bacterial community composition and diversity. The greatest effect of cover cropping on both the phyllosphere community and $P$. syringae abundance was observed early in the growing season. $P$. syringae begins life on leaves as an epiphyte but then must colonize host tissue through stomata or wounds (Misas-Villamil et al. 2013). Disease severity could be lowered if the early establishment and survival of $P$. syringae is compromised.

We found that both RCC and CT-RCC treatments induced a strong shift in the squash phyllosphere microbiota, leading to a distinct community composition in comparison with BS and PC treatments. The largest difference in leaf bacterial community composition associated with RCC was observed early in the growing season. Our results are consistent with previous reports of homogenization of phyllosphere community structure over time; early in the growing season, the leaf microbiota was more diverse and colonization from the soil is likely a strong driver of the phyllosphere microbiome (Copeland et al. 2015). Moreover, shifts in microbial community composition are likely driven by changes in environmental conditions as well as shifts in sources of bacterial migration to the phyllosphere. Cover cropping can directly modify soil abiotic properties such as temperature and moisture and chemical properties (Villamil et al. 2006). Cover cropping also likely influences bacterial dispersal sources, both by promoting colonization by bacteria living on the cover crops themselves as well as through their effects on dispersal from different potential sources such as soils (Bodenhausen et al. 2013), water splash (Butterworth and McCartney 1991), and insects (de Vega and Herrera 2013; Manirajan et al. 2016). Taken together, we hypothesize that such local environmental shifts modify bacterial migration to the phyllosphere early in the growing season. We further speculate that an early shift in the squash phyllosphere may intervene by mechanical transfer from the cover crop, when the young plant goes through the rye mulch.

2016
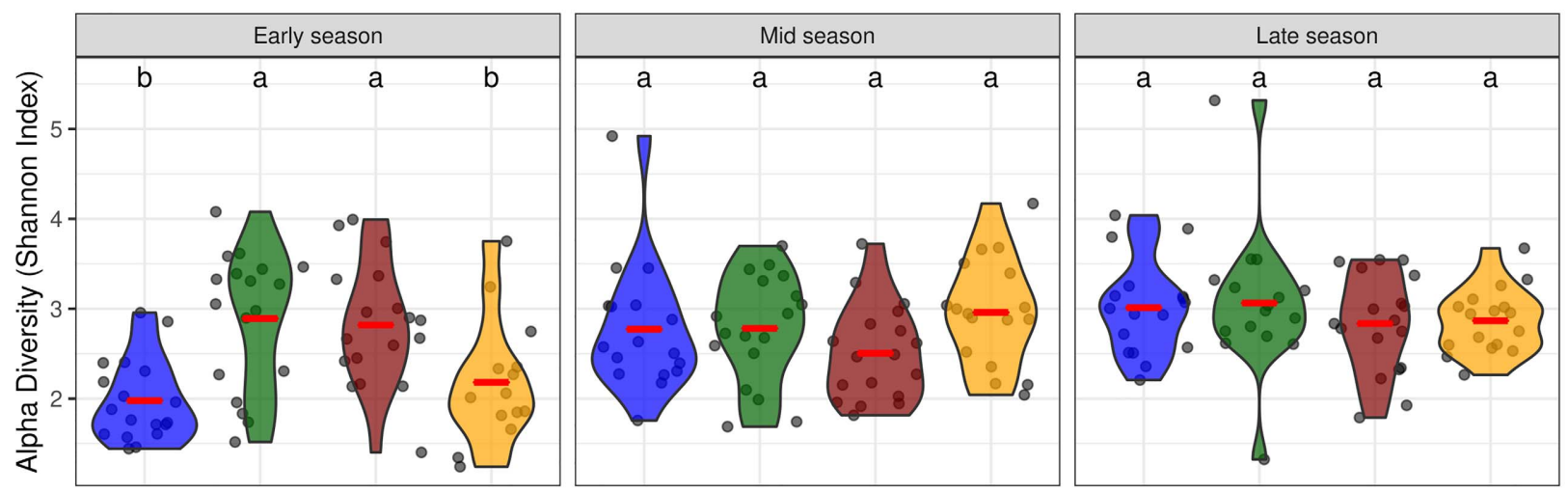

Treatment

2017
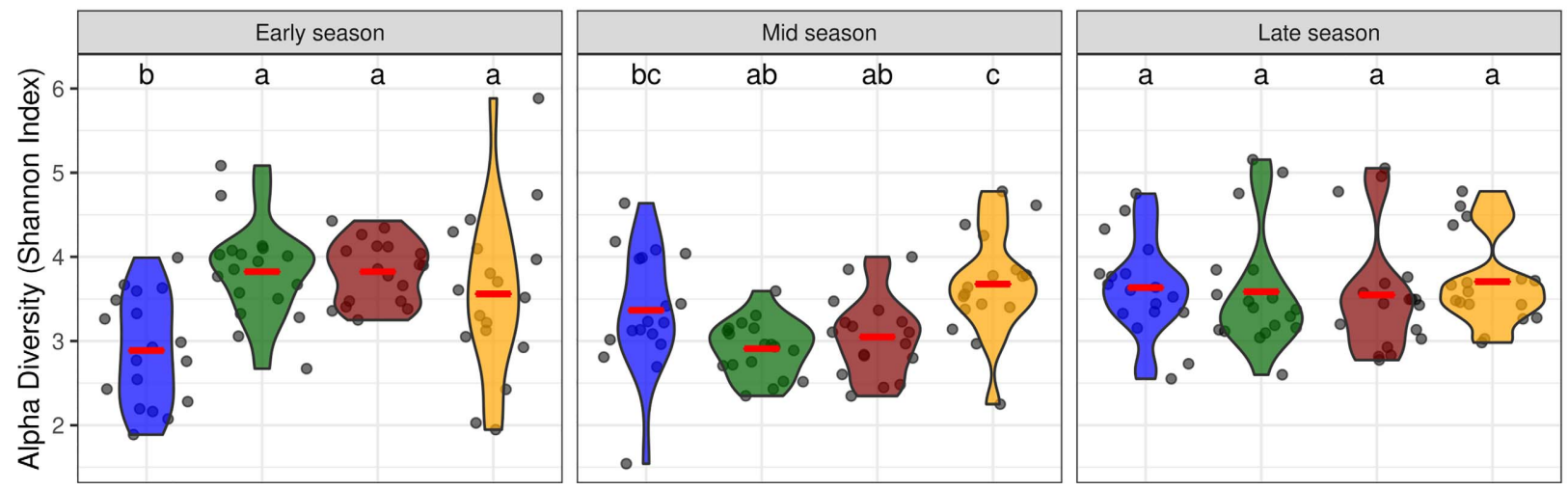

PC

RCC

CT-RCC BS

Fig. 2. $\alpha$ Diversity (Shannon index) of phyllosphere bacterial communities for each treatment and each sampling date during the growing season of years 2016 and 2017. Horizontal red line represents the mean. Blue = plastic cover $(P C)$, green = rye cover crop $(R C C)$, red $=$ chemically terminated rye cover crop (CT-RCC), and yellow = bare soil (BS). Different letter represents significantly different treatments $(P<0.05)$ from a posthoc test (Tukey's honestly significant difference) of a linear model ( $\alpha$ diversity as a function of treatment). 
In addition to the effects of cover cropping on phyllosphere microbial communities, cover cropping treatments also likely influenced environmental conditions, which may explain part of their protective effects against $P$. syringae. Cover crops influence humidity and temperature (Teasdale and Mohler 1993). Moreover, dispersal of epiphytic bacteria is a function of humidity (Lindemann and Upper 1985), and the structure of phyllosphere bacterial communities is significantly influenced by soil temperature (Ren et al. 2015). We observed that soil moisture and temperature varied among cover cropping treatments; soil moisture was higher under RCC and PC relative to BS, and temperatures were elevated under PC relative to other treatments (results not shown). Rye is also known to have allelopathic properties (Schulz et al. 2013), which can influence soil microbiota (Hu et al. 2018). Moreover, rye degradation can lead to decreases in soil pH (Abdollahi and Munkholm 2014) and improve weed control (Barnes and Putnam 1983). During this experiment, no weeds were found for the PC treatment, a few grew in both rye cover treatments, while many were found in the BS treatment (all weeds were manually removed on a routine basis during the growing season). All of these effects of cover cropping on the abiotic and biotic environment could influence early pathogen development and interact with shifts in phyllosphere microbiota to modulate the potential protective effects of cover crops. Furthermore, because P. syringae may likely be seedborne (Hirano and Upper 2000), we inoculated seeds with this pathogen at the time of planting but, in real situations, the effect of cover crops on pathogen populations will also be a function of temporal variation in seed and soil pathogen and microbiota reservoirs.

Previous studies have reported a protective effect against pathogens by phyllosphere microbial diversity per se (Keesing et al. 2010); for example, where increasing diversity of genus Sphingomonas on leaves increased protection against $P$. syringae (Innerebner et al. 2011). We did not find strong evidence for an effect of $\alpha$ diversity on its own to explain the protective effect of cover cropping against $P$. syringae; there were no overall differences in $\alpha$ diversity of phyllosphere bacteria among cover cropping treatments, although RCC did increase diversity in the Early season. To properly test for a protective effect of phyllosphere diversity against $P$. syringae, future studies that directly manipulate diversity while keeping other factors constant will be required; however, our results suggest that it was the composition of bacterial communities and not the diversity of the community per se that could explain the protective effects of cover cropping treatments.

Our results support the hypothesis that RCC could protect against $P$. syringae by promoting the establishment of potential competitors or plant-growth-promoting bacteria (PGPB) on the leaf surface. This included several ASVs belonging to the genus
Early season 2016

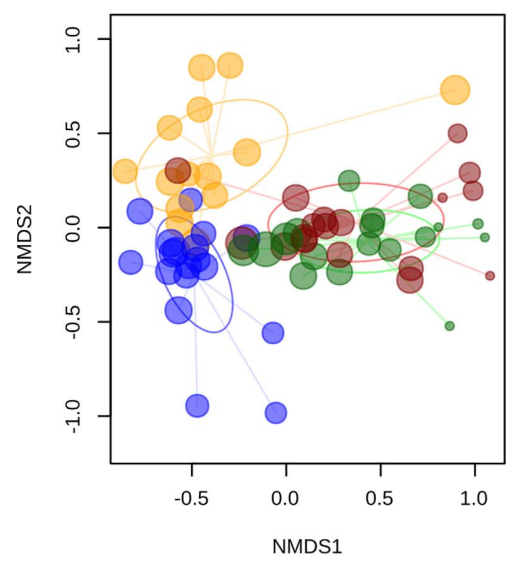

Early season 2017

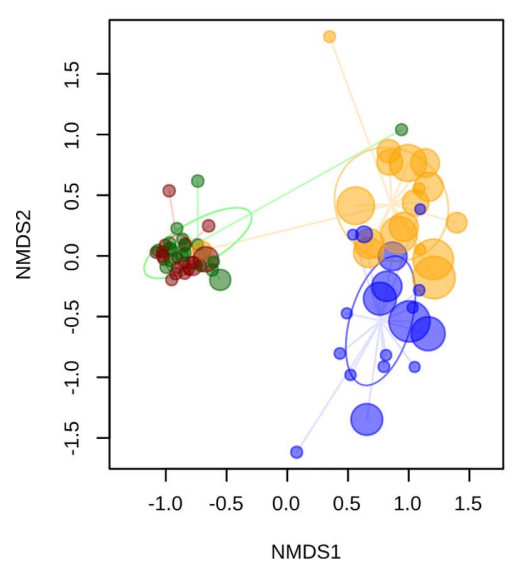

Mid season 2016

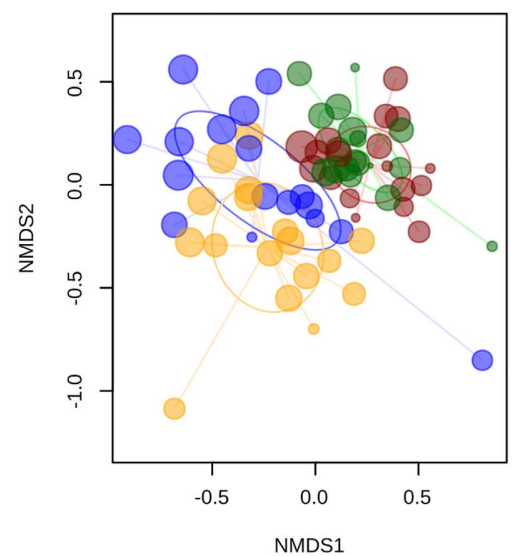

Mid season 2017

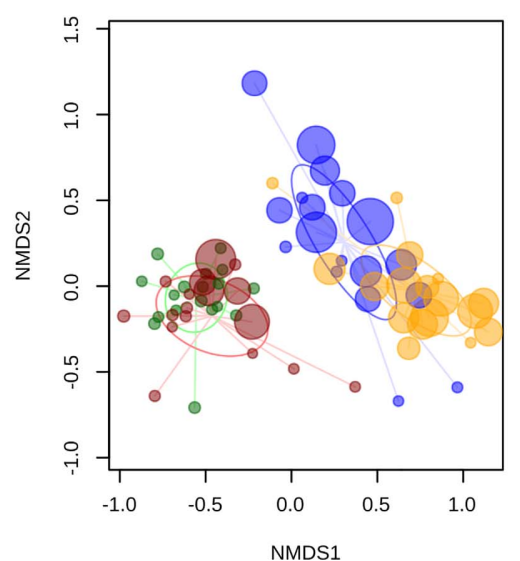

Late season 2016

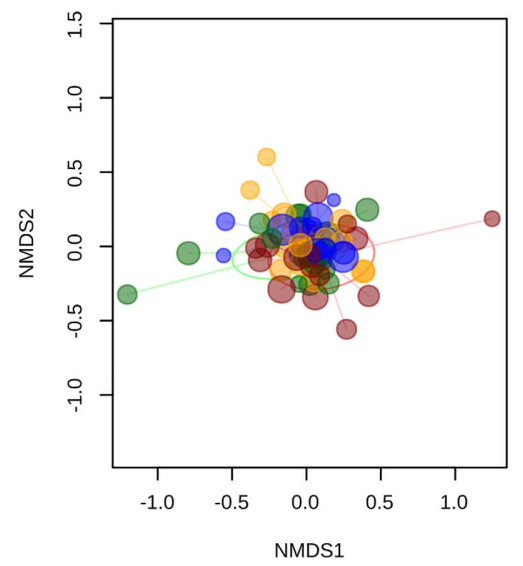

Late season 2017

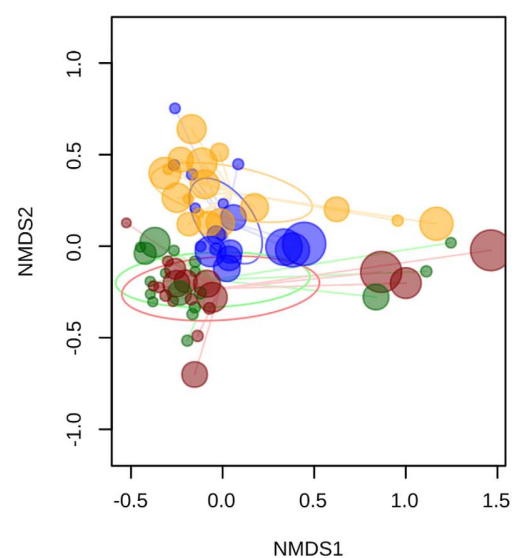

P. syringae $(\log 10($ CFUs))

- 1.11

- 4.575

- 5.19

- 5.006

- 5.688

7.58

Treatment

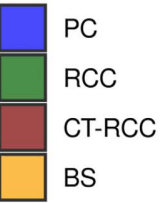
BS (log10(CFUs))

- 1.538

- 1.787

- 1.967

- 3.085

- 4.378

7.641

Fig. 3. Nonmetric multidimensional scaling (NMDS) ordination of bacterial community composition in squash phyllosphere samples from different cover cropping treatments in 2016 and 2017. Each point represents a phyllosphere community; symbol size indicates the abundance of Pseudomonas syringae CFU ( $\log _{10}[\mathrm{CFUs}]$ ) in that sample and colors indicate the cover cropping treatment: blue = plastic cover $(\mathrm{PC})$, green = rye cover crop (RCC), red $=$ chemically terminated rye cover crop $(C T-R C C)$, and yellow $=$ bare soil (BS). 
Sphingomonas, which were more abundant with RCC, especially early in the growing season. Previous studies have demonstrated that Sphingomonas strains protect Arabidopsis against $P$. syringae in a controlled environment (Innerebner et al. 2011). Our findings provide field-based evidence suggesting that the Sphingomonas clade is a potential competitor, given the increased abundance of this genus in the protective RCC treatments. We also found many other taxa preferentially associated with squash under RCC

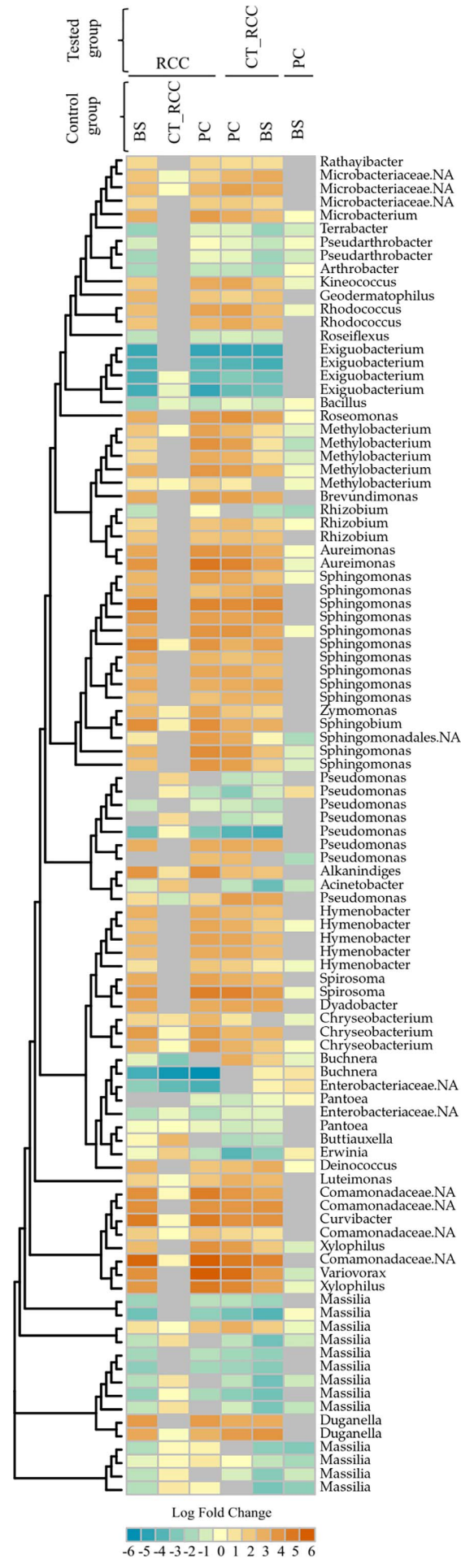

Early season

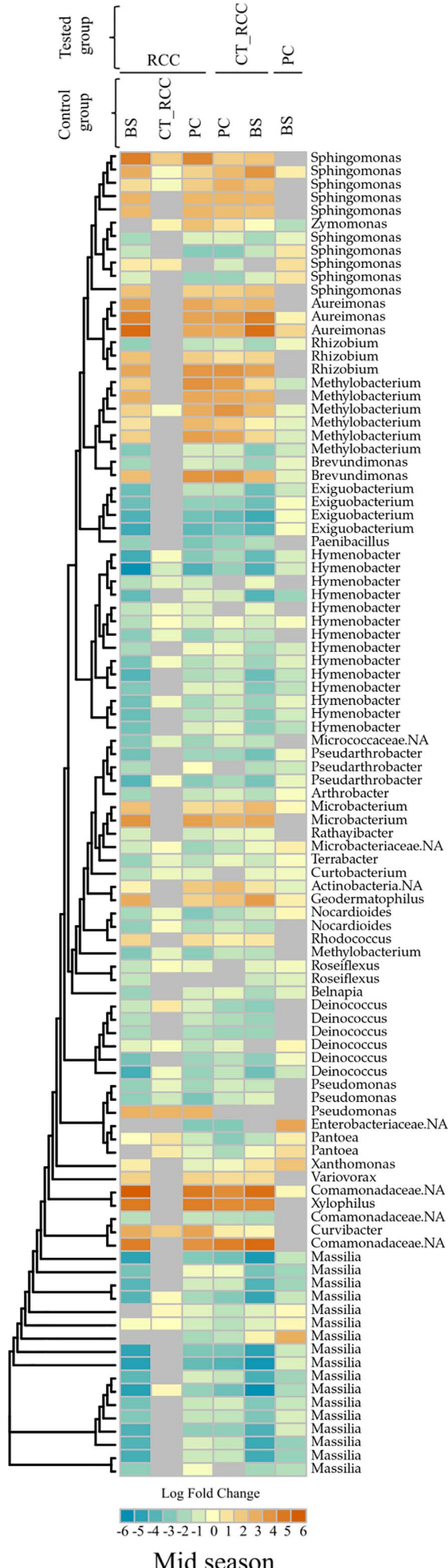

Mid season

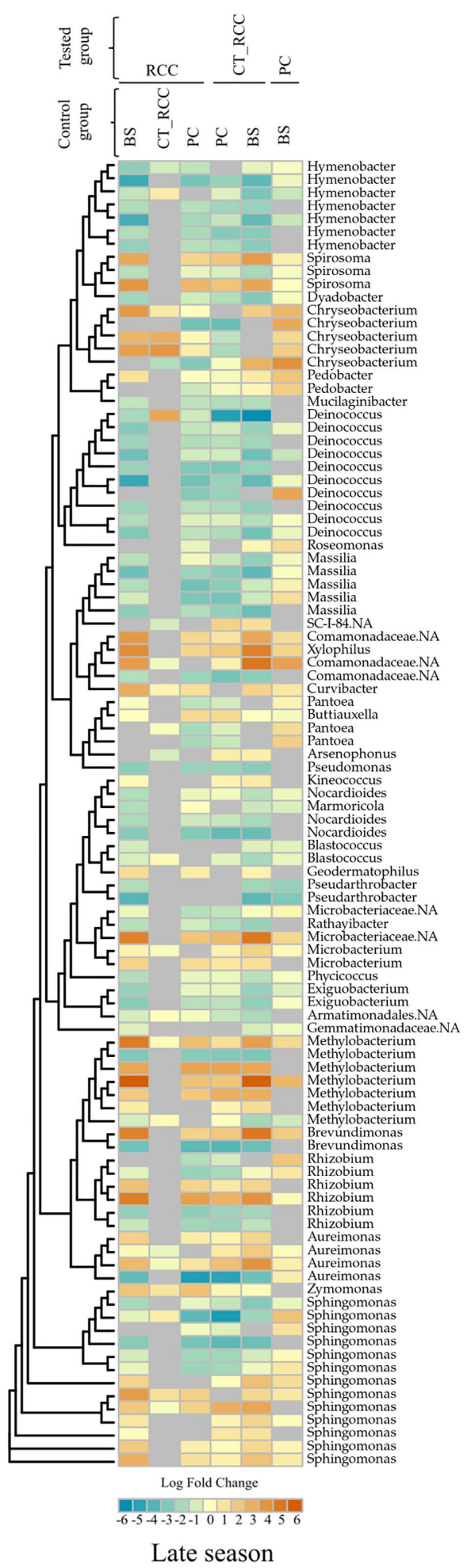

Late season

Fig. 4. Log $_{2}$ fold change (LFC) heatmap of most differentially abundant amplicon sequence variant (ASV) from DeSeq2 analysis for each sampling date of the 2017 samples. For each panel, the left track is the phylogenetic tree from PyNAST alignment of ASV sequences while the right track is the corresponding taxonomic genus name. Each heatmap column is a different contrast between two treatments mentioned in the header as follows: the upper name is the "tested" treatment whereas the lower name is the "control" treatment, meaning that a positive LFC value represents an ASV more abundant for the tested treatment. Gray color represents no LFC for the ASV. Each number on the bottom LFC color scale represents a level of LFC. Tested treatments: plastic cover (PC), rye cover crop (RCC), chemically terminated rye cover crop (CT-RCC), and bare soil (BS). 
treatments, including ASVs belonging to the genus Methylobacterium, which is a phyllosphere-associated clade (Delmotte et al. 2009) known to be an important PGPB in agriculture (Madhaiyan et al. 2006), and nonpathogenic ASVs belonging to the genus Pseudomonas, which has been shown to be an antagonist of the pathogens Erwinia (Cabrefiga Olamendi et al. 2007), tobacco necrosis virus (Maurhofer et al. 1998), and Botrytis cinerea (De Meyer and Höfte 1997). Thus, RCC treatments appear to favor the establishment of potentially plant beneficial bacteria in the phyllosphere. Although our results need to be followed up with more experimental tests to quantify the potential benefits of these genera, the potentially beneficial bacterial ASVs associated with RCC that we have identified are already candidates for exploration of microbiome engineering approaches to directly inoculate protective bacterial strains to protect crops against pathogens (Quiza et al. 2015).

Availability of data and material. The demultiplexed sequence data have been deposited as sequence read archives under BioProject PRJNA705113. The scripts used to perform analyses for the current study are available in a GitHub repository (https://github.com/ RemiMaglione/Science-Communication/tree/main/Article/covercrop-squash-phyllosphere-microbiota-2021).

\section{ACKNOWLEDGMENTS}

We thank K. Bisaillon, P.-O. Hebert, and J.-B. Leducq for their help in the field, in the lab, with analysis, and for discussions.

\section{LITERATURE CITED}

Abanda-Nkpwatt, D., Krimm, U., Schreiber, L., and Schwab, W. 2006. Dual antagonism of aldehydes and epiphytic bacteria from strawberry leaf surfaces against the pathogenic fungus Botrytis cinerea in vitro. BioControl 51:279-291.

Abawi, G. S., and Widmer, T. L. 2000. Impact of soil health management practices on soilborne pathogens, nematodes and root diseases of vegetable crops. Appl. Soil Ecol. 15:37-47.

Abdollahi, L., and Munkholm, L. J. 2014. Tillage system and cover crop effects on soil quality: I. Chemical, mechanical, and biological properties. Soil Sci. Soc. Am. J. 78:262-270.

Andrews, J. H. 1992. Biological control in the phyllosphere. Annu. Rev. Phytopathol. 30:603-635.

Bakker, M. G., Acharya, J., Moorman, T. B., Robertson, A. E., and Kaspar, T. C. 2016. The potential for cereal rye cover crops to host corn seedling pathogens. Phytopathology 106:591-601.

Barnes, J. P., and Putnam, A. R. 1983. Rye residues contribute weed suppression in no-tillage cropping systems. J. Chem. Ecol. 9:1045-1057.

Bender, C. L., and Cooksey, D. A. 1986. Indigenous plasmids in Pseudomonas syringae pv. tomato: Conjugative transfer and role in copper resistance. J. Bacteriol. 165:534-541.

Bodenhausen, N., Horton, M. W., and Bergelson, J. 2013. Bacterial communities associated with the leaves and the roots of Arabidopsis thaliana. PLoS One 8:e56329.

Bray, J. R., and Curtis, J. T. 1957. An ordination of the upland forest communities of southern Wisconsin. Ecol. Monogr. 27:325-349.

Bushnell, B. 2014. BBTools software package. https://jgi.doe.gov/data-andtools/bbtools/

Butterworth, J., and McCartney, H. A. 1991. The dispersal of bacteria from leaf surfaces by water splash. J. Appl. Bacteriol. 71:484-496.

Cabrefiga, J., Bonaterra, A., and Montesinos, E. 2007. Mechanisms of antagonism of Pseudomonas fluorescens EPS62e against Erwinia amylovora, the causal agent of fire blight. Int. Microbiol. 10:123-132.

Callahan, B. J., McMurdie, P. J., Rosen, M. J., Han, A. W., Johnson, A. J. A., and Holmes, S. P. 2016. DADA2: High-resolution sample inference from Illumina amplicon data. Nat. Methods 13:581-583.

Caporaso, J. G., Bittinger, K., Bushman, F. D., DeSantis, T. Z., Andersen, G. L., and Knight, R. 2010a. PyNAST: A flexible tool for aligning sequences to a template alignment. Bioinformatics 26:266-267.
Caporaso, J. G., Kuczynski, J., Stombaugh, J., Bittinger, K., Bushman, F. D., Costello, E. K., Fierer, N., Peña, A. G., Goodrich, J. K., Gordon, J. I., Huttley, G. A., Kelley, S. T., Knights, D., Koenig, J. E., Ley, R. E., Lozupone, C. A., McDonald, D., Muegge, B. D., Pirrung, M., Reeder, J., Sevinsky, J. R., Turnbaugh, P. J., Walters, W. A., Widmann, J., Yatsunenko, T., Zaneveld, J., and Knight, R. 2010b. QIIME allows analysis of highthroughput community sequencing data. Nat. Methods 7:335-336.

Chelius, M. K., and Triplett, E. W. 2001. The diversity of Archaea and Bacteria in association with the roots of Zea mays L. Microb. Ecol. 41:252-263.

Collins, H. P., Alva, A., Boydston, R. A., Cochran, R. L., Hamm, P. B., McGuire, A., and Riga, E. 2006. Soil microbial, fungal, and nematode responses to soil fumigation and cover crops under potato production. Biol. Fertil. Soils 42:247-257.

Copeland, J. K., Yuan, L., Layeghifard, M., Wang, P. W., and Guttman, D. S. 2015. Seasonal community succession of the phyllosphere microbiome. Mol. Plant-Microbe Interact. 28:274-285.

Dabney, S., Delgado, J., and Reeves, D. 2001. Using winter cover crops to improve soil and water quality. Commun. Soil Sci. Plant Anal. 32:1221-1250.

Delmotte, N., Knief, C., Chaffron, S., Innerebner, G., Roschitzki, B., Schlapbach, R., von Mering, C., and Vorholt, J. A. 2009. Community proteogenomics reveals insights into the physiology of phyllosphere bacteria. Proc. Natl. Acad. Sci. U.S.A. 106:16428-16433.

De Meyer, G., and Höfte, M. 1997. Salicylic acid produced by the Rhizobacterium Pseudomonas aeruginosa 7NSK2 induces resistance to leaf infection by Botrytis cinerea on bean. Phytopathology 87: 588-593.

de Vega, C., and Herrera, C. M. 2013. Microorganisms transported by ants induce changes in floral nectar composition of an ant-pollinated plant. Am. J. Bot. 100:792-800.

Fawcett, J., Mitchell, T., Rogers, J., and Rossiter, L. 2017. On-farm cover crop trials. Farm Prog. Rep. 2016.

Fawcett, J., Sievers, J., Roush, W., and Lang, B. 2015. On-farm cover crop trials. Farm Prog. Rep. 2014.

Fawell, J., and Nieuwenhuijsen, M. J. 2003. Contaminants in drinking water: Environmental pollution and health. Br. Med. Bull. 68:199-208.

Gloor, G. 2016. CoDaSeq: Analyzing HTS using compositional data analysis. F1000 Res. 5-1285.

Haegeman, B., Hamelin, J., Moriarty, J., Neal, P., Dushoff, J., and Weitz, J. S. 2013. Robust estimation of microbial diversity in theory and in practice. ISME J. 7:1092-1101.

Hall, J. K., Hartwig, N. L., and Hoffman, L. D. 1984. Cyanazine losses in runoff from no-tillage corn in "living" and dead mulches vs. unmulched, conventional tillage. J. Environ. Qual. 13:105-110.

Hartman, K., van der Heijden, M. G. A., Wittwer, R. A., Banerjee, S., Walser, J.-C., and Schlaeppi, K. 2018. Cropping practices manipulate abundance patterns of root and soil microbiome members paving the way to smart farming. Microbiome 6:14.

Hirano, S. S., and Upper, C. D. 2000. Bacteria in the leaf ecosystem with emphasis on Pseudomonas syringae-A pathogen, ice nucleus, and epiphyte. Microbiol. Mol. Biol. Rev. 64:624-653.

Hooks, C. R. R., Wang, K.-H., Ploeg, A., and McSorley, R. 2010. Using marigold (Tagetes spp.) as a cover crop to protect crops from plantparasitic nematodes. Appl. Soil Ecol. 46:307-320.

Hu, L., Robert, C. A. M., Cadot, S., Zhang, X., Ye, M., Li, B., Manzo, D., Chervet, N., Steinger, T., van der Heijden, M. G. A., Schlaeppi, K., and Erb, M. 2018. Root exudate metabolites drive plant-soil feedbacks on growth and defense by shaping the rhizosphere microbiota. Nat. Commun. 9:2738.

Innerebner, G., Knief, C., and Vorholt, J. A. 2011. Protection of Arabidopsis thaliana against leaf-pathogenic Pseudomonas syringae by Sphingomonas strains in a controlled model system. Appl. Environ. Microbiol. 77:32023210.

Keesing, F., Belden, L. K., Daszak, P., Dobson, A., Harvell, C. D., Holt, R. D., Hudson, P., Jolles, A., Jones, K. E., Mitchell, C. E., Myers, S. S., Bogich, T., and Ostfeld, R. S. 2010. Impacts of biodiversity on the emergence and transmission of infectious diseases. Nature 468:647-652.

Kembel, S. W., Cowan, P. D., Helmus, M. R., Cornwell, W. K., Morlon, H., Ackerly, D. D., Blomberg, S. P., and Webb, C. O. 2010. Picante: R tools for integrating phylogenies and ecology. Bioinformatics 26:1463-1464.

Lenth, R., Singmann, H., Love, J., Buerkner, P., and Herve, M. 2018. emmeans: Estimated marginal means, aka least-squares means. R Package version 1.3. https://rdrr.io/cran/emmeans/

Lindemann, J., Arny, D. C., and Upper, C. D. 1984. Epiphytic populations of Pseudomonas syringae pv. syringae on snap bean and nonhost plants and 
the incidence of bacterial brown spot disease in relation to cropping patterns. Phytopathology 74:1329-1333.

Lindemann, J., and Suslow, T. V. 1987. Competition between ice nucleationactive wild type and ice nucleation-deficient deletion mutant strains of Pseudomonas syringae and Pseudomonas fluorescens biovar I and biological control of frost injury on strawberry blossoms. Phytopathology 77: 882-886.

Lindemann, J., and Upper, C. D. 1985. Aerial dispersal of epiphytic bacteria over bean plants. Appl. Environ. Microbiol. 50:1229-1232.

Lindow, S. E., and Brandl, M. T. 2003. Microbiology of the phyllosphere. Appl. Environ. Microbiol. 69:1875-1883.

Love, M. I., Huber, W., and Anders, S. 2014. Moderated estimation of fold change and dispersion for RNA-seq data with DESeq2. Genome Biol. 15: 550.

Lu, Y.-C., Watkins, K. B., Teasdale, J. R., and Abdul-Baki, A. A. 2000. Cover crops in sustainable food production. Food Rev. Int. 16:121-157.

Madhaiyan, M., Suresh Reddy, B. V., Anandham, R., Senthilkumar, M., Poonguzhali, S., Sundaram, S. P., and Sa, T. 2006. Plant growthpromoting Methylobacterium induces defense responses in groundnut (Arachis hypogaea L.) compared with rot pathogens. Curr. Microbiol. 53: 270-276.

Manirajan, B. A., Ratering, S., Rusch, V., Schwiertz, A., Geissler-Plaum, R., Cardinale, M., and Schnell, S. 2016. Bacterial microbiota associated with flower pollen is influenced by pollination type, and shows a high degree of diversity and species-specificity. Environ. Microbiol. 18:5161-5174.

Martin, M. 2011. Cutadapt removes adapter sequences from high-throughput sequencing reads. EMBnet. J. 17:10-12.

Maurhofer, M., Reimmann, C., Schmidli-Sacherer, P., Heeb, S., Haas, D., and Défago, G. 1998. Salicylic acid biosynthetic genes expressed in Pseudomonas fluorescens Strain P3 improve the induction of systemic resistance in tobacco against Tobacco necrosis virus. Phytopathology 88:678-684.

McMurdie, P. J., and Holmes, S. 2013. phyloseq: An R package for reproducible interactive analysis and graphics of microbiome census data. PLoS One 8:e61217.

Misas-Villamil, J. C., Kolodziejek, I., Crabill, E., Kaschani, F., Niessen, S., Shindo, T., Kaiser, M., Alfano, J. R., and van der Hoorn, R. A. L. 2013. Pseudomonas syringae pv. syringae uses proteasome inhibitor syringolin a to colonize from wound infection sites. PLoS Pathog 9:e1003281.

Nearing, J. T., Douglas, G. M., Hayes, M., MacDonald, J., Desai, D., Allward, N., Jones, C. M. A., Wright, R., Dhanani, A., Comeau, A. M., and Langille, M. G. I. 2021. Microbiome differential abundance methods produce disturbingly different results across 38 datasets. BioRxiv 2021.05.10.443486.

Oerke, E.-C. 2006. Crop losses to pests. J. Agric. Sci. 144:31-43.

Oksanen, J., Kindt, R., Legendre, P., O'Hara, B., Stevens, M. H. H., Oksanen, M. J., and Suggests, M. 2007. The vegan package. Community Ecol. Package 10:631-637.

Pieterse, C. M. J., Zamioudis, C., Berendsen, R. L., Weller, D. M., Van Wees, S. C. M., and Bakker, P. A. H. M. 2014. Induced systemic resistance by beneficial microbes. Annu. Rev. Phytopathol. 52:347-375.
Price, M. N., Dehal, P. S., and Arkin, A. P. 2010. FastTree 2-Approximately maximum-likelihood trees for large alignments. PLoS One 5:e9490.

Quast, C., Pruesse, E., Yilmaz, P., Gerken, J., Schweer, T., Yarza, P., Peplies, J., and Glöckner, F. O. 2013. The SILVA ribosomal RNA gene database project: Improved data processing and web-based tools. Nucleic Acids Res. 41:D590-D596.

Quiza, L., St-Arnaud, M., and Yergeau, E. 2015. Harnessing phytomicrobiome signaling for rhizosphere microbiome engineering. Front. Plant Sci. 6:507.

R Core Team. 2013. R: A Language and Environment for Statistical Computing. R Foundation for Statistical Computing, Vienna, Austria.

Redford, A. J., Bowers, R. M., Knight, R., Linhart, Y., and Fierer, N. 2010. The ecology of the phyllosphere: Geographic and phylogenetic variability in the distribution of bacteria on tree leaves. Environ. Microbiol. 12:2885-2893.

Ren, G., Zhu, C., Alam, M. S., Tokida, T., Sakai, H., Nakamura, H., Usui, Y., Zhu, J., Hasegawa, T., and Jia, Z. 2015. Response of soil, leaf endosphere and phyllosphere bacterial communities to elevated $\mathrm{CO}_{2}$ and soil temperature in a rice paddy. Plant Soil 392:27-44.

Ritpitakphong, U., Falquet, L., Vimoltust, A., Berger, A., Métraux, J.-P., and L'Haridon, F. 2016. The microbiome of the leaf surface of Arabidopsis protects against a fungal pathogen. New Phytol. 210:1033-1043.

Savary, S., Willocquet, L., Pethybridge, S. J., Esker, P., McRoberts, N., and Nelson, A. 2019. The global burden of pathogens and pests on major food crops. Nat. Ecol. Evol. 3:430-439.

Schulz, M., Marocco, A., Tabaglio, V., Macias, F. A., and Molinillo, J. M. G. 2013. Benzoxazinoids in rye allelopathy-From discovery to application in sustainable weed control and organic farming. J. Chem. Ecol. 39:154-174.

Stirzaker, R. J., and White, I. 1995. Amelioration of soil compaction by a cover-crop for no-tillage lettuce production. Aust. J. Agric. Res. 46:553-568.

Teasdale, J. R. 1996. Contribution of cover crops to weed management in sustainable agricultural systems. J. Prod. Agric. 9:475-479.

Teasdale, J. R., and Mohler, C. L. 1993. Light transmittance, soil temperature, and soil moisture under residue of hairy vetch and rye. Agron. J. 85:673-680.

Van Wees, S. C., Van der Ent, S., and Pieterse, C. M. 2008. Plant immune responses triggered by beneficial microbes. Curr. Opin. Plant Biol. 11: 443-448.

Villamil, M. B., Bollero, G. A., Darmody, R. G., Simmons, F. W., and Bullock, D. G. 2006. No-till corn/soybean systems including winter cover crops. Soil Sci. Soc. Am. J. 70:1936-1944.

Wickham, H. 2016. ggplot2: Elegant Graphics for Data Analysis. Use R! Springer, Cham, Switzerland.

Xin, X.-F., Kvitko, B., and He, S. Y. 2018. Pseudomonas syringae: What it takes to be a pathogen. Nat. Rev. Microbiol. 16:316-328.

Yilmaz, P., Parfrey, L. W., Yarza, P., Gerken, J., Pruesse, E., Quast, C., Schweer, T., Peplies, J., Ludwig, W., and Glöckner, F. O. 2014. The SILVA and "All-species Living Tree Project (LTP)" taxonomic frameworks. Nucleic Acids Res. 42:D643-D648. 